\title{
Association Between Built Space and Cognition in Humans: A Scoping Review
}

\author{
Prachi Dave ${ }^{1}$, S Sudha ${ }^{2}$,Vijaya Raghavan ${ }^{1}$, P Poornachandrika ${ }^{2}$
}

\begin{abstract}
Background: Various built environment characteristics have been shown to have varied effects on mental health. There is evidence for a positive relationship between certain $\mathrm{BE}$ characteristics and mental disorders. The talk on cognition is inevitable in any argument on mental health. This review looks at various $\mathrm{BE}$ characteristics and cognition and their interrelationship.
\end{abstract}

Materials and Methods: A Boolean search of PubMed articles with keywords like 'built environment', 'built spaces', 'green spaces', greenspace,'neighbourhood environment' and 'attention', 'concentration', 'cognition', 'cognitive function' and 'cognitive disorders' was carried out and relevant articles were chosen by two individual investigators.

Results: 26 articles were chosen for the study, of which 22 were cross-sectional, 1 longitudinal, 1 cohort, 1 retrospective and 1 prospective study.

Conclusion: There is a lot of evidence for the positive correlation between green spaces and cognitive performance. Greenness, biodiversity, walkability has been investigated to show a beneficial effect on cognition, especially attention restoration. Longitudinal studies that include persons with comorbidities will help as better evidences for the above positive correlation.

Keywords: Built Environment, Built Spaces, Green Spaces, Walkability, Cognition, Attention

Running Title: Built Space and Cognition in Humans
Address for Correspondence: Dr Vijaya Raghavan, Consultant Psychiatrist - Research, Schizophrenia Research Foundation, Chennai. E-mail: vijayaraghavan@scarfindia.org

How to Cite this Article: Dave P, Sudha S, Vijaya Raghavan, Poornachandrika P. Association between built space and cognition in humans: A scoping review. Indian Journal of Mental Health and Neurosciences.2020;3(1): pp 23-35

B uilt environment includes all man-made structures that constitute the space that we live and move in, like buildings, parks, malls, temples etc. ${ }^{1}$ Built environment has shown to have a huge impact on different dimensions of human life, including thoughts, emotion and behaviour. ${ }^{2}$ With the advent of studies on human-environment interactions, more research has been directed towards specific environmental characteristics and their impact on human life and health. The results from such studies have helped the humans to build sustainable and healthy environments which are ergonomically designed to improve productivity, reduce stress and facilitate behavioural changes.

Cognition is one of the core components of mental health and illness. Cognition is defined as the mental action or process of acquiring knowledge and understanding through thought, experience, and the senses. ${ }^{3}$ The six domains of cognition are - complex attention, executive function, learning and memory, language, perceptualmotor function, and social cognition. ${ }^{4}$ Cognition is needed not only for the complex intellectual processes

${ }^{1}$ Schizophrenia Research Foundation, Chennai, Tamilnadu, India. ${ }^{2}$ Institute of Mental Health, Madras Medical College, Chennai, Tamilnadu, India. 
such as abstract thinking and problem solving, but also for the normal functioning in day-to-day activities.

Cognition is affected in many of the mental disorders such as schizophrenia leading to various cognitive deficits including poor attention and executive function. ${ }^{5}$ These cognitive deficits have a huge impact on the functional recovery in patients with mental disorders leading to poor quality of life and high caregiver burden. The emerging fields of cognitive neuroscience and cognitive psychology are trying to understand the basics of cognition and translation of these findings to improve cognition in normal people and remedial interventions for patients with cognitive deficits.

Various characteristics of the built environment have been found to have associations with different mental health dimensions including cognition.6 For example, the association of green spaces with lower incidence of stress and incidence of greater anomie in people residing in high-rise buildings highlight the importance of the built spaces in determining human emotion and behaviour. ${ }^{7}$ Similarly, a properly designed built environment is helpful in the development of healthier children and healthier communities. ${ }^{8}$ Biophilic environments have shown to have a positive impact on mental health. Biophilia is a term coined by Erich Fromm that points to intense love for nature. Biophilic design in architecture involves connectivity to nature through direct and indirect means. ${ }^{9}$

There is modest research on the relationship between built environment characteristics and cognition and fewer reviews on the same. However, there are no holistic reviews that address questions on all built environment characteristics and their impact on cognition. Hence, in this review, we have attempted to synthesize and summarize the studies that have examined the associations between built environment and cognition in humans.

\section{MATERIAL \& METHODS}

Scoping reviews are those that enable us to map out the key concepts via research by using available evidence. ${ }^{10}$ Through sequential steps, the scoping review process maps and synthesizes key underlying concepts in a particular field to identify disparities in existing knowledge. ${ }^{11}$ The information extracted for this scoping review was, the author, sample of study, type of study, instruments used and major findings. Two research investigators screened a total of 453 articles for its relevance by title. This brought the number down to 62 articles. These 62 articles were subjected to another screening process. This time the articles were selected based on their relevance from the content of the abstract, leaving us with 32 articles. NCBI PUBMED was the search engine used to extract the relevant articles from MEDLINE Database. Initially, free and broadened search was encouraged, to pick out relevant articles. Key words such as: 'built environment \& mental health', 'built environment \& cognition', were used to identify the articles and draw out the common keywords. For built environment keywords such as "green space", "built space", "green environment" and "green space" were used. And for cognition, keywords such as "cognitive enhancement", "attention", "cognitive disorders" and "concentration" were used. Eventually, a combination of keywords: ("built space" OR "built environment" OR "green space" OR "green environment") AND (cognition OR "cognitive enhancement" OR "cognitive disorder" OR attention OR concentration) were searched in the PubMed Advanced search Builder in the field of 'text word'. Language filter was applied for articles in 'English'. All possible dates of publication were considered.

\section{RESULTS}

With the above-mentioned search terms, after adding filters for English language and excluding reviews, we got 453 articles for review. On further scrutiny 62 articles were selected based on relevance of title and 32 based on abstract. Finally, we included 26 articles in our scoping review (Table 1). The studies mostly included samples from the general population, few samples from previous cohorts, school-going children and college students and public health registry. We had 22 crosssectional studies, 1 retrospective, 1 prospective and 1 cohort study. The articles were grouped according to the cognitive function that was studied in the research, and we found most of the articles pertaining to attention and some on cognition in a broader sense. The studies also had heterogeneity in the built environment characteristics that were studied but the most common being greenness. 
Table 1 Summary findings from the studies included on built environment and cognition

\begin{tabular}{|c|c|c|c|c|}
\hline Author, Year & Type of study & Sample & Tools & Key findings \\
\hline $\begin{array}{l}\text { Wood et al., } \\
2018^{12}\end{array}$ & $\begin{array}{l}\text { Crosssectional } \\
\text { survey }\end{array}$ & $\begin{array}{l}\text { People entering the } \\
12 \text { parks located in } \\
\text { Bradford ( } \mathrm{n}=128)\end{array}$ & $\begin{array}{l}\text { NEST (Natural } \\
\text { Environment Scoring } \\
\text { Tool), } 21 \text { point } \\
\text { Attention Restoration } \\
\text { Theory questionnaire. }\end{array}$ & $\begin{array}{l}\text { There is a positive } \\
\text { association between site } \\
\text { facilities and biodiversity. } \\
\text { Restorative benefit is } \\
\text { predicted by biodiversity, } \\
\text { which explained } 43 \% \text { of } \\
\text { the variance in restorative } \\
\text { benefit across the parks, } \\
\text { with minimal input from } \\
\text { other variables. } \\
\text { The benefits accrued } \\
\text { through access to green } \\
\text { space were unrelated to } \\
\text { age, gender, and ethnic } \\
\text { background. }\end{array}$ \\
\hline Lin et al., $2020^{13}$ & $\begin{array}{l}\text { Cross sectional } \\
\text { study }\end{array}$ & 40 college students & $\begin{array}{l}\text { Emotiv EPOC (ex- } \\
\text { cess post exercise ox- } \\
\text { ygen consumption) + } \\
\text { EEG (electroenceph- } \\
\text { alogram) headset to } \\
\text { dynamically measure } \\
6 \text { neuralemotional pa- } \\
\text { rameters: engagement, } \\
\text { valence, meditation, } \\
\text { frustration, focus, and } \\
\text { excitement. } \\
\text { ANCOVA to analyse } \\
\text { pre-test and post-test } \\
\text { variants }\end{array}$ & $\begin{array}{l}\text { Walking in urban green } \\
\text { space is more favourable } \\
\text { for stress reduction. } \\
\text { Sitting in urban green } \\
\text { space is better for attention } \\
\text { restoration. }\end{array}$ \\
\hline $\begin{array}{l}\text { Mygind et al., } \\
2018^{14}\end{array}$ & $\begin{array}{l}\text { Cross sectional } \\
\text { study }\end{array}$ & $\begin{array}{l}47 \text { children aged } \\
10-12 \text { years in } \\
\text { school }\end{array}$ & $\begin{array}{l}\text { Stress response by } \\
\text { Heart-rate monitor. } \\
\text { Cognitive performance } \\
\text { by D2 test. }\end{array}$ & $\begin{array}{l}\text { No evidence to support } \\
\text { that the children's cognitive } \\
\text { performances were } \\
\text { improved in the natural } \\
\text { environments compared to } \\
\text { classrooms. }\end{array}$ \\
\hline $\begin{array}{l}\text { Reeves et al., } \\
2019^{15}\end{array}$ & $\begin{array}{l}\text { Cross sectional } \\
\text { study }\end{array}$ & $\begin{array}{l}36 \text { participants } \\
\text { through social } \\
\text { corporate } \\
\text { responsibility }\end{array}$ & $\begin{array}{l}\text { HR (heart rate) and } \\
\text { EEG (electroencepha- } \\
\text { logram), DASS (de- } \\
\text { pression anxiety stress } \\
\text { scale) Holmes and Rake } \\
\text { stress inventory, Nature } \\
\text { relatedness scale }\end{array}$ & 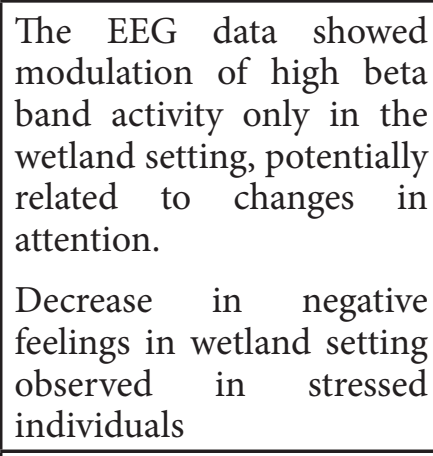 \\
\hline $\begin{array}{l}\text { de keijzeret al., } \\
2018^{16}\end{array}$ & $\begin{array}{l}\text { Cross sectional } \\
\text { study }\end{array}$ & $\begin{array}{l}6506 \text { participants' } \\
\text { data from the } \\
\text { Whitehall II cohort }\end{array}$ & $\begin{array}{l}\text { Normalized Difference } \\
\text { Vegetation Index; } \\
\text { N D V I R e a s o n in g, } \\
\text { verbal fluency, short } \\
\text { term memory. }\end{array}$ & $\begin{array}{l}\text { Higher residential } \\
\text { surrounding greenness } \\
\text { was associated with slower } \\
\text { cognitive decline over a } \\
10 \text {-y follow-up period in } \\
\text { the Whitehall II cohort of } \\
\text { civil servants. }\end{array}$ \\
\hline
\end{tabular}




\begin{tabular}{|c|c|c|c|c|}
\hline $\begin{array}{l}\text { Zijlema et al., } \\
2017^{19}\end{array}$ & $\begin{array}{l}\text { Cross sectional } \\
\text { study }\end{array}$ & $\begin{array}{l}\text { Between } \\
\mathrm{n}=1493 \text { and } \\
\mathrm{n}=1602 \text { participants } \\
\text { for the current } \\
\text { analyses out of } 1628 \\
\text { participants who } \\
\text { completed the CTT }\end{array}$ & $\begin{array}{l}\text { Colour trails test (CTT) } \\
\text { for assessing cognitive } \\
\text { function }\end{array}$ & $\begin{array}{l}\text { Each } 100 \mathrm{~m} \text { increase in } \\
\text { residential distance to } \\
\text { NOE was associated with } \\
\text { a longer CTT completion } \\
\text { time of } 1.50 \% \text { (95\% CI } \\
0.13,2.89) \text {. } \\
\text { Higher loneliness and } \\
\text { more air pollution worries } \\
\text { were associated with } \\
\text { longer CTT completion } \\
\text { time, while higher social } \\
\text { cohesion and better } \\
\text { mental health were related } \\
\text { to shorter CTT completion } \\
\text { time. }\end{array}$ \\
\hline $\begin{array}{l}\text { Dadvand et al., } \\
2018^{20}\end{array}$ & $\begin{array}{l}\text { Cross sectional } \\
\text { study }\end{array}$ & $\begin{array}{l}253 \text { school children } \\
\text { without special } \\
\text { needs }\end{array}$ & $\begin{array}{l}\text { Normalized differ- } \\
\text { ence vegetation index } \\
\text { (NDVI), n back test, } \\
\text { Attentional Network } \\
\text { Task ANT, 3D MRI } \\
\text { (magnetic resonance } \\
\text { imaging) }\end{array}$ & $\begin{array}{l}\text { Brain regions with larger } \\
\text { volumes in urban children } \\
\text { with higher lifelong } \\
\text { exposure to residential } \\
\text { surrounding greenness, } \\
\text { especially those involved in } \\
\text { cognitive functions. }\end{array}$ \\
\hline $\begin{array}{l}\text { Markevych et } \\
\text { al., } 2018^{21}\end{array}$ & $\begin{array}{l}\text { Cross sectional } \\
\text { study }\end{array}$ & $\begin{array}{l}66,823 \text { children, all } \\
\text { beneficiaries of the } \\
\text { health insurance } \\
\text { company AOK } \\
\text { PLUS and born } \\
\text { between } 2000 \text { and } \\
2004\end{array}$ & $\begin{array}{l}\text { MODIS (moderate } \\
\text { resolution imaging } \\
\text { spectroradiometer) } \\
\text { Normalized Differ- } \\
\text { ence Vegetation Index } \\
\text { (NDVI) }\end{array}$ & $\begin{array}{l}\text { A } 0.1 \text {-unit increase in } \\
\text { NDVI decreased the } \\
\text { relative risk of ADHD by a } \\
\text { factor of } 0.82 \text {. } \\
\text { An increase of PM10 and } \\
\mathrm{NO} 2 \text { by } 10 \mu \mathrm{g} / \mathrm{m} 3 \text { raised } \\
\text { the relative risk of ADHD } \\
\text { by a factor of } 1.97 \text { and } 1.32 \text {, } \\
\text { respectively. }\end{array}$ \\
\hline $\begin{array}{l}\text { Ward et al., } \\
2016^{22}\end{array}$ & $\begin{array}{l}\text { Cross sectional } \\
\text { study }\end{array}$ & $\begin{array}{l}108 \text { participants } \\
\text { aged } 11-14 \text { years } \\
\text { from three inter- } \\
\text { mediate schools } \\
\text { in Auckland, New } \\
\text { Zealand }\end{array}$ & $\begin{array}{l}\text { Computerised neuro- } \\
\text { cognitive testing was } \\
\text { conducted using CNS } \\
\text { (central nervous sys- } \\
\text { tem) Vital Signs }\end{array}$ & $\begin{array}{l}\text { No significant association } \\
\text { between green space } \\
\text { exposure and cognition. }\end{array}$ \\
\hline $\begin{array}{l}\text { Tiesler et al } \\
2013^{23}\end{array}$ & $\begin{array}{l}\text { Crosssectional } \\
\text { study }\end{array}$ & $\begin{array}{l}1,932-10 \text { year } \\
\text { old children with } \\
\text { complete exposure } \\
\text { to green space. }\end{array}$ & $\begin{array}{l}\text { Behavioural problems } \\
\text {-Strengths \& Difficul- } \\
\text { ties Questionnaire. } \\
\text { Access to green space - } \\
\text { distance to nearest } \\
\text { green space. }\end{array}$ & $\begin{array}{l}\text { Poor access to urban green } \\
\text { spaces associated with } \\
\text { behavioural problems, } \\
\text { hyperactivity/inattention } \\
\text { problems. More significant } \\
\text { in male children. } \\
\text { Access to forests unrelated } \\
\text { to children's mental health. }\end{array}$ \\
\hline $\begin{array}{l}\text { Bodin et al., } \\
2015^{24}\end{array}$ & $\begin{array}{l}\text { Cross sectional } \\
\text { study }\end{array}$ & $\begin{array}{l}\text { 2612persons of } \\
\text { Malmo, Sweden. }\end{array}$ & $\begin{array}{l}\text { A residential environ- } \\
\text { ment survey }\end{array}$ & $\begin{array}{l}\text { Access to a quiet side } \\
\text { (facing yard, water or green } \\
\text { space) was associated with } \\
\text { reduced risk of annoyance } \\
\text { \& concentration problems. } \\
\text { Bedroom window facing } \\
\text { the same environment was } \\
\text { associated to better sleep }\end{array}$ \\
\hline
\end{tabular}




\begin{tabular}{|c|c|c|c|c|}
\hline $\begin{array}{l}\text { Donovan et al., } \\
2019^{25}\end{array}$ & $\begin{array}{l}\text { Longitudinal } \\
\text { study }\end{array}$ & $\begin{array}{l}923 \text { eligible children } \\
\text { born in } 1998 \text { in New } \\
\text { Zealand who were } \\
\text { selected using IDI } \\
\text { (Integrated Data } \\
\text { Infrastructure). }\end{array}$ & $\begin{array}{l}\text { Exposure to green } \\
\text { space estimated using } \\
\text {-Normalized Difference } \\
\text { Vegetation Index } \\
\text { (NVDI). }\end{array}$ & $\begin{array}{l}\text { Increasing lifetime } \\
\text { exposure greenness } \\
\text { reduces risk of ADHD. }\end{array}$ \\
\hline $\begin{array}{l}\text { Sharp et al., } \\
2019^{26}\end{array}$ & Cohort study & $\begin{array}{l}\text { A cohort of } 190 \\
\text { children ( } 96 \text { with } \\
\text { ADHD). }\end{array}$ & $\begin{array}{l}\text { The } 190 \text { children had } \\
\text { a range of neigh- } \\
\text { bour-hood\& familial } \\
\text { factors ascertained \& } \\
\text { had repeated clinical } \\
\text { assessments over an } \\
\text { average of } 2.5 \text { years at } \\
\text { a U.S. research centre. } \\
\text { Using mixed model } \\
\text { regression, they found } \\
\text { an association between } \\
\text { neighbourhood wealth } \\
\& \text { inattentiveness. } \\
\text { Using transactional } \\
\text { model, they checked } \\
\text { if familial processes, } \\
\text { influence the results. }\end{array}$ & $\begin{array}{l}\text { Children in relatively } \\
\text { affluent neighbourhoods } \\
\text { showed improvement } \\
\text { with age in inattention, } \\
\text { largely independent of } \\
\text { variation in a wide range } \\
\text { of familial factors such } \\
\text { as family conflict, family } \\
\text { educational/economic } \\
\text { status. } \\
\text { Children living in less } \\
\text { affluent neighbourhoods } \\
\text { showed clinical deteriora- } \\
\text { tion only if the family } \\
\text { had high levels of conflict } \\
\text { or if the parents were of } \\
\text { lower economic/education } \\
\text { status. }\end{array}$ \\
\hline $\begin{array}{l}\text { Allen et al., } \\
2016^{27}\end{array}$ & $\begin{array}{l}\text { Cross sectional } \\
\text { study }\end{array}$ & $\begin{array}{l}24 \text { Participants who } \\
\text { spent } 6 \text { full working } \\
\text { days (9am }-5 \mathrm{pm}) .\end{array}$ & $\begin{array}{l}\text { On different days, they } \\
\text { were exposed to IEQ } \\
\text { (indoor environmen- } \\
\text { tal quality) conditions } \\
\text { representative of Con- } \\
\text { ventional [highcon- } \\
\text { centrations of volatile } \\
\text { organic compounds } \\
\text { (VOCs)] andGreen } \\
\text { (low concentrations of } \\
\text { VOCs) office buildings } \\
\text { in the United States. } \\
\text { Additional conditions } \\
\text { simulated a green } \\
\text { building with a high } \\
\text { outdoor air ventilation } \\
\text { rate (labelled Green+) } \\
\text { and artificiallyelevated } \\
\text { carbon dioxide (CO2) } \\
\text { levels independent of } \\
\text { ventilation. }\end{array}$ & $\begin{array}{l}\text { On average, cognitive } \\
\text { scores were } 61 \% \text { higher } \\
\text { on the Green building day } \\
\text { and } 101 \% \text { higher on the } \\
\text { two Green+ building days } \\
\text { than on the Conventional } \\
\text { building day ( } \mathrm{p}<0.0001 \text { ). } \\
\text { VOCs and CO2 were } \\
\text { independently associated } \\
\text { with cognitive scores. }\end{array}$ \\
\hline Gao et al., $2019^{28}$ & $\begin{array}{l}\text { Cross sectional } \\
\text { study }\end{array}$ & $\begin{array}{l}120 \text { healthy } \\
\text { Chineseparticipants } \\
\text { voluntarily } \\
\text { Recruitedvia } \\
\text { 'WeChat' }\end{array}$ & $\begin{array}{l}\text { Randomly divided } \\
20 \text { participants in a } \\
\text { group,exposed to one } \\
\text { of the 6environments } \\
\text { via Virtual Reality. } \\
\text { EEG (electroencepha- } \\
\text { logram) - to measure } \\
\text { physiological stress. }\end{array}$ & $\begin{array}{l}\text { Restorative effects on } \\
\text { attentional fatigue and } \\
\text { negative mood using VR } \\
\text { (virtual reality) was seen. } \\
\text { Partly open green (POG) } \\
\text { space has a significant } \\
\text { effect on negative mood } \\
\text { regulation. }\end{array}$ \\
\hline
\end{tabular}




\begin{tabular}{|c|c|c|c|c|}
\hline & & & $\begin{array}{l}\text { Profile of Mood } \\
\text { States(POMS-SF) } \\
\text { scale - used to measure } \\
\text { psychological stress. }\end{array}$ & $\begin{array}{l}\text { A strong positive } \\
\text { correlation was seen } \\
\text { between preference of an } \\
\text { environment and positive } \\
\text { mood. } \\
\text { Blue \& green spaces have } \\
\text { restorative effect }\end{array}$ \\
\hline $\begin{array}{l}\text { Amoly et al., } \\
2014^{29}\end{array}$ & $\begin{array}{l}\text { Cross sectional } \\
\text { study }\end{array}$ & $\begin{array}{l}2,111 \text { school } \\
\text { children ( } 7-10 \\
\text { years of age) from } \\
36 \text { schools in } \\
\text { Barcelona in } 2012 .\end{array}$ & $\begin{array}{l}\text { Data on: Time spent } \\
\text { in green spaces and } \\
\text { beaches and Strengths } \\
\text { and Difficulties Ques- } \\
\text { tionnaires (SDQ) from } \\
\text { parents \& ADHD/ } \\
\text { DSM-IV questionnaires } \\
\text { from teachers. NDVI } \\
\text { used to define green- } \\
\text { ness. }\end{array}$ & 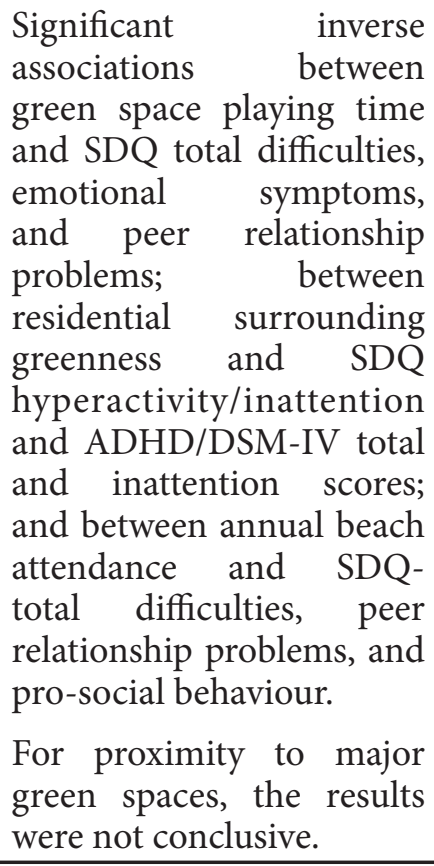 \\
\hline $\begin{array}{l}\text { Amicone et al., } \\
2018^{30}\end{array}$ & $\begin{array}{l}\text { Cross sectional } \\
\text { study }\end{array}$ & $\begin{array}{l}82 \text { primary school } \\
\text { children between } \\
\text { 4thand 5th grade in } \\
\text { middle urban area } \\
\text { of Rome, Italy. }\end{array}$ & $\begin{array}{l}\text { Study } 1 \text { - morning } \\
\text { recesstime, team play } \\
\text { was allowed in natural } \\
\text { environment. } \\
\text { Study 2- afternoon } \\
\text { recesstime, team play } \\
\text { was allowed in built } \\
\text { environment. }\end{array}$ & $\begin{array}{l}\text { An increase in sustained } \\
\text { and selective attention after } \\
\text { the natural environment } \\
\text { condition and a decrease } \\
\text { after the built environment } \\
\text { break. } \\
\text { Higher scores in perceived } \\
\text { restorativeness were } \\
\text { registered after the natural } \\
\text { (vs. built) environment } \\
\text { condition. } \\
\text { Team standardized } \\
\text { playtime and individual } \\
\text { free play recess in natural } \\
\text { environment (vs. built) } \\
\text { support pupils attention } \\
\text { restoration during both } \\
\text { morning and afternoon } \\
\text { school times, as well as their } \\
\text { perceived restorativeness } \\
\text { of the recess environment. }\end{array}$ \\
\hline $\begin{array}{l}\text { Cherrie et al., } \\
2018^{31}\end{array}$ & $\begin{array}{l}\text { Retrospective } \\
\text { study }\end{array}$ & $\begin{array}{l}281 \text { participants } \\
\text { of Lothian birth } \\
\text { cohort. }\end{array}$ & $\begin{array}{l}\text { At mean age } 78-\text { a } \\
\text { standalone question- } \\
\text { naire was adminis- } \\
\text { tered. At mean ages } \\
11,70 \& 76-\text { the Mo- } \\
\text { ray House Test no.12 } \\
\text { (MHT) was adminis- } \\
\text { tered to test cognition. }\end{array}$ & $\begin{array}{l}\text { Neighbourhood provision } \\
\text { of public parks from } \\
\text { childhood through to } \\
\text { adulthood may help slow } \\
\text { down the rate of cognitive } \\
\text { decline in later life. }\end{array}$ \\
\hline
\end{tabular}




\begin{tabular}{|c|c|c|c|c|}
\hline & & & $\begin{array}{l}\text { Other genetic,demo- } \\
\text { graphic and socio-eco- } \\
\text { nomic information was } \\
\text { collected via question- } \\
\text { naires. }\end{array}$ & $\begin{array}{l}\text { The association between } \\
\text { childhood and adulthood } \\
\text { park availability and } \\
\text { change in the Moray House } \\
\text { Test Score from age } 7 \text { to } 76 \\
\text { was strongest for women, } \\
\text { those without an APOE e4 } \\
\text { allele. }\end{array}$ \\
\hline $\begin{array}{l}\text { Dadvand et al., } \\
2015^{32}\end{array}$ & $\begin{array}{l}\text { Cross sectional } \\
\text { study }\end{array}$ & $\begin{array}{l}2,593 \text { schoolchildren } \\
\text { in the } 2 \text { nd to } 4 \text { th } \\
\text { grades ( } 7-10 \text { years) } \\
\text { of } 36 \text { primary } \\
\text { schools in } \\
\text { Barcelona, Spain } \\
(2012-2013) \text {. }\end{array}$ & $\begin{array}{l}\text { Greenness - NDVI } \\
\text { (normalized difference } \\
\text { vegetation index) and } \\
\text { cognitive development } \\
\text { computerized atten- } \\
\text { tional network test. }\end{array}$ & $\begin{array}{l}\text { Beneficial association } \\
\text { between exposure to } \\
\text { green space and cognitive } \\
\text { development among } \\
\text { schoolchildren that } \\
\text { was partly mediated by } \\
\text { reduction in exposure to } \\
\text { air pollution. }\end{array}$ \\
\hline $\begin{array}{l}\text { Crous-Bou et } \\
\text { Al., } 2020^{33}\end{array}$ & $\begin{array}{l}\text { Cross-sectional } \\
\text { study. }\end{array}$ & $\begin{array}{l}958 \text { middle- } \\
\text { aged, cognitively } \\
\text { unimpaired } \\
\text { subjects, many of } \\
\text { them offspring } \\
\text { of Alzheimer's } \\
\text { Dementia (AD) } \\
\text { patients. }\end{array}$ & $\begin{array}{l}\text { To study airpollution- } \\
\text { European Study of Co- } \\
\text { horts for Air Pollution } \\
\text { Effects (ESCAPE). } \\
\text { Greenness- NDVI } \\
\text { Cognitive develop- } \\
\text { ment-Memory Binding } \\
\text { Test (MBT), Wechsler } \\
\text { Adult Intelligence } \\
\text { Scale. (WAIS)-IV, } \\
\text { Preclinical Alzheimer } \\
\text { Cognitive Composite } \\
\text { (PACC), MRI Scan }\end{array}$ & $\begin{array}{l}\text { No significant associations } \\
\text { were observed between } \\
\text { urban environmental } \\
\text { exposures and the cognitive } \\
\text { composite }(>0.1) \text {. } \\
\text { Higher exposure to air } \\
\text { pollutants, but not noise, } \\
\text { was associated with lower } \\
\text { cortical thickness in brain } \\
\text { regions known to be } \\
\text { affected by AD, especially } \\
\text { NO2 and PM10. } \\
\text { Increasing greenness } \\
\text { indicators was associated } \\
\text { with greater thickness in } \\
\text { these same areas }\end{array}$ \\
\hline $\begin{array}{l}\text { Dadvand et al., } \\
2017^{34}\end{array}$ & $\begin{array}{l}\text { Prospective } \\
\text { study }\end{array}$ & $\begin{array}{l}\text { Based on data from } \\
2 \text { well established } \\
\text { population based } \\
\text { birth cohorts in } \\
\text { Spain. }\end{array}$ & $\begin{array}{l}\text { Greenness- NDVICon- } \\
\text { ners' Kiddie Continu- } \\
\text { ous PerformanceTest } \\
\text { (K-CPT ) at } 4-5 \text { years } \\
(\mathrm{n}=888) \text { and the At- } \\
\text { tentional Network } \\
\text { Task (ANT) at } 7 \text { years } \\
(\mathrm{n}=987) .\end{array}$ & $\begin{array}{l}\text { Children who had more } \\
\text { exposure to green space } \\
\text { had lesser omission errors } \\
\text { in the K-CPT and ANT. } \\
\text { Associations with } \\
\text { residential surrounding } \\
\text { tree cover also were close } \\
\text { to the null, or were negative } \\
\text { (for ANT HRT-SE) but no } \\
\text { statistically significant. }\end{array}$ \\
\hline $\begin{array}{l}\text { De Vries et al., } \\
2016^{35}\end{array}$ & $\begin{array}{l}\text { Cross sectional } \\
\text { study }\end{array}$ & $\begin{array}{l}\text { 6621 participants. } \\
\text { Dutch speaking } 18- \\
24 \text { year olds. }\end{array}$ & $\begin{array}{l}\text { Health data were de- } \\
\text { rived from a nationally } \\
\text { representative survey } \\
\text { (NEMESIS-2). Com- } \\
\text { posite International } \\
\text { Diagnostic Interview } \\
\text { (CIDI), version 3.0. } \\
\text { Mental Health Invento- } \\
\text { ry-5 (MHI-5). } \\
\text { Dutch Land Use da- } \\
\text { tabase, LGN6 - assess } \\
\text { green space. }\end{array}$ & $\begin{array}{l}\text { Associations were } \\
\text { generally stronger for blue } \\
\text { space than for green space, } \\
\text { with ORs up to } 0.74 \text { for a } \\
10 \% \text {-point increase. }\end{array}$ \\
\hline
\end{tabular}




\begin{tabular}{|c|c|c|c|c|}
\hline $\begin{array}{l}\text { Besser et al., } \\
2018^{36}\end{array}$ & $\begin{array}{l}\text { Cross sectional } \\
\text { study }\end{array}$ & $\begin{array}{l}4539 \text { participant's } \\
\text { from the Multi- } \\
\text { Ethnic Study of } \\
\text { Atherosclerosis }\end{array}$ & $\begin{array}{l}\text { Cognitive meas- } \\
\text { ure-MESA's (multi } \\
\text { ethnic study of athero- } \\
\text { sclerosis) Exam dedi- } \\
\text { cated to residences or } \\
\text { retail was calculated by } \\
\text { dividing the Residen- } \\
\text { tial/retail area by the } \\
\text { total buffer area }\end{array}$ & $\begin{array}{l}\text { Increasing social } \\
\text { destination density, walking } \\
\text { destination density, and } \\
\text { intersection density } \\
\text { were associated with } \\
\text { worse cognition, whereas } \\
\text { increasing proportion of } \\
\text { land dedicated to retail } \\
\text { was associated with better } \\
\text { processing speed. } \\
\text { Certain characteristics } \\
\text { in dense urban } \\
\text { environments may have } \\
\text { a disproportionately } \\
\text { negative association with } \\
\text { cognition in vulnerable } \\
\text { populations. } \\
\text { APOE } 2 \text { carriers may be } \\
\text { more susceptible to the } \\
\text { potentiallybeneficial effects } \\
\text { of denser neighbourhood } \\
\text { BEs on cognition. }\end{array}$ \\
\hline $\begin{array}{l}\text { Kodama et al., } \\
2010^{37}\end{array}$ & $\begin{array}{l}\text { Cross-sectional } \\
\text { study }\end{array}$ & $\begin{array}{l}18 \text { patients with } \\
\text { traumatic brain } \\
\text { injury and } 18 \text { normal } \\
\text { controls }\end{array}$ & $\begin{array}{l}\text { WAIS III (Wechsler's } \\
\text { Adult Scale Intel- } \\
\text { ligence),P } 300 \text { - } \\
\text { Trail-making test. }\end{array}$ & $\begin{array}{l}\text { TBI (traumatic brain } \\
\text { injury) patients showed a } \\
\text { smaller amplitude of P } 300 \\
\text { in the red environment } \\
\text { than control subjects. } \\
\text { In patients, P300 latency } \\
\text { was significantly shorter } \\
\text { in the green environment } \\
\text { than in the other colour } \\
\text { environment. } \\
\text { Rehabilitation intended to } \\
\text { improve selective attention } \\
\text { or information processing } \\
\text { speed might be more } \\
\text { effective if performed in a } \\
\text { low anxiety environment } \\
\text { (i.e., a green environment). }\end{array}$ \\
\hline
\end{tabular}

\section{DISCUSSION}

\section{Built environment and cognition}

$\mathrm{Ng}$ et al., observed that there was a positive and significant association between land use mix diversity, walkability and repeatable Battery for the Assessment of Neuropsychological Status (RBANS) z score, immediate and delayed memory, recall, visuospatial and constructional ability but not attention. ${ }^{18}$ This is in contrast to the attention restoration theory of Kaplan. Allen et al., showed better cognitive scores in people having 1 or 2 green building days in their workspace. ${ }^{27}$ Ward et al., found no association between greenspace exposure and cognition as measured by computerized
CNS vital signs..22 Besser et al., found association between denser urban environments and worse cognition.36Dadvand et al., 2015 had shown better cognitive development in children in green spaces. ${ }^{32}$

de Keijzer et al., showed slower cognitive decline in people exposed to greater residential surrounding greenness over 10 years; especially in the reasoning and fluency tests and not in short term memory. ${ }^{16}$ Mediation analysis of three potential mediators (physical activity, social support, air pollution) for this association suggested no mediation. Provision of public parks in childhood and early adulthood is associated with lesser cognitive decline according to Cherrie and colleagues. ${ }^{31} \mathrm{Wu}$ et al., (2017) had shown that living in areas with higher 
land use mix is associated with $30 \%$ decreased odds of cognitive impairment. ${ }^{17}$ No significant association has been found between PACC and exposure to urban environments according to Crousbou and colleagues. ${ }^{33}$

Besser found that the association between natural environment characteristics and cognitive decline is associated with APOE2 and not with APOE3 or $4 .{ }^{36}$ According to Dadvand et al., there is an association between lifetime greenspace exposure and larger brain volumes, especially the right and left prefrontal cortex.20Crousbou et al., have shown greenspace to be associated with higher cortical thickness in the brain and air pollution the converse. ${ }^{33}$

All studies on cognition have been cross sectional studies or studies taking samples from larger cohorts wherein there is a possibility for bias. The results of studies on greenspace and cognition are not comparable as there is heterogeneity in testing of cognition. Studies on cognitive decline have no matching for possible physical and psychiatric comorbidities that might increase the risk of cognitive impairment. Overall, the studies lack investigation on long term memory, executive functioning, social cognition or perceptual motor functioning. The very intrusive nature of an objective 'cognitive function' testing after brief exposure to any environment is not devoid of the confounder of performance anxiety and bias.

There might be a complex interaction of multiple factors in the association between green spaces and cognition. Oliver Mytton et al., have found a positive correlation between green space and physical activity and physical activity. ${ }^{38}$ This mediates better cognition and neuroplasticity through epigenetic mechanisms. ${ }^{39}$ Thus, physical activity may be an important mediator in the effects of green space on cognition. Various studies have proven green spaces to reduce state anxiety and cause stress recovery. It is also known well that stress and anxiety have a dual role on cognition ${ }^{40}$ but higher the anxiety, lower the cognitive performance. ${ }^{41}$ Probable mediators for attention restoration in greenspace like anxiety in green environment versus other built spaces and their effect on EEG high beta band and P 300 latency offer insights into mechanisms behind such restorative benefits as expressed by the above studies. 28

\section{Attention restoration and green space}

A study by Lin et al., observes 6 neutral emotional parameters. Out of the 6, only valence and meditation show consistent results. ${ }^{13}$ The "valence" \& "meditation" parameters are higher in participants who were walking $(\mathrm{M}=0.71 \& 0.58)$ in green space, when compared to participants who were sitting $(\mathrm{M}=0.63 \& 0.33)$ in green space. Whereas, the "focus" parameter showed a small contrary result of $(\mathrm{M}=0.55)$ in the sitting group which is higher than $(M=0.42)$ in the walking group. Gao et al in their study subjected the participants to 6 different environments through VR. It was observed that there was no significant impact of the exposed environments on restoration of attention fatigue or positive mood. "open-green space" environment showed restorative effects of negative mood.

Both of the studies have been carried out in a short time frame. A better understanding of the same can be achieved by conducting long term studies. In the study by Gao et al., VR technology was used to expose the subjects to green spaces. Adopting technology to study the effect of green environment may not give authentic results when compared to studies that use natural environments. ${ }^{28}$

\section{Attention restoration \& natural/built environment}

Wood et al., used the attention restoration theory (ART) questionnaire in their cross-sectional study. It was found out that, out of all factors (such as site facilities), biodiversity played an important role in attention restoration by $43 \% .{ }^{12}$ In another study, Amicone et al., divided the children into 2 groups. The first group that was given recess break in the natural environment, showed improvement in "sustained and selectiveattention". The students' score in Bell's test was higher after the recess $(M=32.61)$ than when compared to before the recess $(M=31.85)$. The other group of students, who were given recess breaks in the built environment, showed no significant results. ${ }^{30}$

The above 2 studies were carried out with a local residential population. Therefore, it cannot be referred to show any ethnic, social or cultural differences. To get more holistic results, a much more random population can be taken and tools that meet standard criteria can be used.

\section{Inattention/hyperactivity/ADHD and green space}

A cross sectional studyshowed that children's behavioural problems were associated with their access to green space. ${ }^{23}$ Using the Strengths \& difficulties questionnaire, the behavioural problems were assessed. Children who had more behavioural problems such as mainly: hyperactivity or inattention were not exposed much to 
green space. It was also found that there is no association between exposure to forest area and behaviour. Donovan et al., found out in their longitudinal study that, children who lived in rural areas, after 2 years of age were less likely to develop ADHD. Their odds ratio was found out to be 0.607 . Exposure to rural living or NDVI only for the first 2 years of life did not decrease the risk of developing ADHD. ${ }^{25}$ Amoly et al., in their crosssectional study observed that there was a significant inverse relationship between exposure to green spaces and the scores in SDQ \& ADHD questionnaire (DSM IV). ${ }^{29}$

In the 3 studies above, Amoly et al., \& Tiesler et al., use the Strengths \& Difficulties Questionnaire to study the behaviour and hence the findings can be compared. ${ }^{23,29}$ Amoly et al., \& Donovan et al., used normalized difference vegetation index (NDVI) to measure greenness which gives more uniform findings. 25,29 The drawbacks of Amoly et al., \& Tiesler et al., are that they are cross-sectional studies. They need more evidence to back up their results. ${ }^{23}{ }^{29}$ These 2 studies can be supported using Donovan et al. Their study is a longitudinal study. This way the findings of each study can be contrasted and compared to establish a link and further give it more value.

\section{ADHD and neighbourhood environment}

Among 190 children in a cross-sectional study, 96 were diagnosed with ADHD/inattention. $47 \%$ of these children showed improvement of symptoms with age. ${ }^{26}$ This study also shows that children in neighbourhoods that are rich and affluent showed less clinical symptoms of ADHD.

This cohort-study has studied the influence of neighbourhood on children with ADHD. While doing so, it is known that neighbourhood factors are vast and cannot be controlled. Sharp et al have considered only a few baseline factors and hence the results do not give us a better understanding of the study. No standard tools were used to measure these factors. This study has to be associated with others that study familial and neighbourhood factors to give its results more evidence and value.

\section{Selective attention and green space}

Kodama et al. in their study showed that, in patients with TBI, P300 latency was significantly shorter in the green environment than in darkness $(p<0.01){ }^{37}$ Rehabilitation intended to improve selective attention or information processing speed might be more effective if performed in a low anxiety environment (i.e., a green environment).

\section{ADHD and pollution}

Markevych et al., in their study observed that a 0.1 increase in NDVI decreased the risk of ADHD by a factor of 0.82 . They also saw that an increase in PM10 \& NO2 raised the risk of ADHD by a factor of 1.97.21 In the study, no proper information on SES and psychopathology of the family to assess risk of ADHD were found out. Understanding information of these factors will help in achieving a more holistic view of the results.

\section{Concentration and quiet side}

In another study, Bodin et al., observed that most participants were annoyed due to the noise and pollution from the traffic. 24 Those who had bedrooms facing a green space had less concentration problems as they had proper sleep. This study lacks sociodemographic information. Further on, they use selfreport questionnaires to assess the level of pollution. This can bring the possibility of bias.

\section{Conclusion}

The role played by spatial cognition in the various cognitive domains is of paramount importance in assessing the impact of environment on cognition. The study of such interactions is essential for building cognitively enhancing residential and workspaces. Architectural models that incorporate research findings on spatial cognition could be utilized for better living spaces. ${ }^{42}$ Virtual reality implementation to simulate natural environments in an urban setting is a new idea in ergonomics to enhance cognitive work performance. ${ }^{43}$ Such studies also provide insight into the psychopathology behind cognitive disorders, the role of environmental factors in the development of a cognitive disorder and ways to mitigate disability in the same. The close relationship between cognition and behaviour warrants a liaison with environment-behaviour studies (EBS) in this regard. ${ }^{44}$

There is a lot of evidence for the positive correlation between green spaces and cognitive performance. ${ }^{45}$ The most common dimension studied is attention. The heterogeneity in testing of cognition makes meta-analysis difficult. The possible mediators of positive effects of greenspace and built environment 
characteristics on cognition need further investigation. Longitudinal studies that include persons with comorbidities will help as better evidence for the above positive correlation. Greenness, biodiversity, walkability have all been investigated to show a beneficial effect on cognition, especially attention restoration.

ACKNOWLEDGEMENT: None

\section{SOURCE OF FUNDING:}

This research has not received specific financial grant from any funding agency in the public, commercialor not-for-profit sectors

\section{CONFLICT OF INTEREST :}

The authors have declared no conflict ofinterest with respect to the research, authorship,and/or publication of this article.

\section{REFERENCES}

1. Crowe N. Nature and the idea of a man-made world: an investigation into the evolutionary roots of form and order in the built environment: MIT press; 1995.

2. Evans GW. The built environment and mental health. Journal of Urban Health. 2003;80(4):536-555

3. Greeno JG, Collins AM, Resnick LB. Cognition and learning. Handbook of educational psychology. 1996;77:1546

4. Sachdev PS, Blacker D, Blazer DG, et al. Classifying neurocognitive disorders: the DSM-5 approach. Nature Reviews Neurology. 2014;10(11):634

5. Bowie CR, Harvey PD. Cognitive deficits and functional outcome in schizophrenia. Neuropsychiatric Disease and Treatment. 2006;2(4):531

6. Firdaus G. Built environment and health outcomes: Identification of contextual risk factors for mental wellbeing of older adults. Rhetoric and Public Affairs. 2017;42(1):62-77

7. Roe JJ, Thompson CW, Aspinall PA, et al. Green space and stress: evidence from cortisol measures in deprived urban communities. International Journal of Environmental Research and Public Health. 2013;10(9):40864103

8. Perdue WC, Stone LA, Gostin LO. The built environment and its relationship to the public's health: the legal framework. American Journal of Public Health. 2003;93(9):1390-1394

9. Gautam A. Biophilic Design in Architecture. International Journal of Engineering Research and Technology. 2017;6(3):120-124

10. Mays N, Roberts E, Popay J. Synthesising research evidence. Studying the organisation and delivery of health services: Research Methods. 2001;220

11. Arksey H, O’Malley L. Scoping studies: towards a methodological framework. International Journal of Social Research Methodology. 2005;8(1):19-32

12. Wood E, Harsant A, Dallimer M, et al. Not all green space is created equal: biodiversity predicts psychological restorative benefits from urban green space. Frontiers in Psychology. 2018;9:2320

13. Lin W, Chen Q, Jiang M, et al. Sitting or Walking? Analyzing the Neural Emotional Indicators of Urban Green Space Behavior with Mobile EEG. Journal of Urban Health. 2020:1-13

14. Mygind L, Stevenson MP, Liebst LS, et al. Stress response and cognitive performance modulation in classroom versus natural environments: A quasi-experimental pilot study with children. International Journal of Environmental Research and Public Health. 2018;15(6):1098 
15. Reeves JP, Knight AT, Strong EA, et al. The application of wearable technology to quantify health and wellbeing co-benefits from urban wetlands. Frontiers in Psychology. 2019;10:1840

16. de Keijzer C, Tonne C, Basagaña X, et al. Residential surrounding greenness and cognitive decline: a 10-year follow-up of the Whitehall II cohort. Environmental Health Perspectives. 2018;126(7):077003

17. Wu Y, Prina A, Jones A, et al. Medical Research Council Cognitive Function and Ageing Study Collaboration. The built environment and cognitive disorders: Results from the cognitive function and ageing study II. American Journal of Preventive Medicine. 2017;53:25-32

18. Ng TP, Nyunt MSZ, Shuvo FK, et al. The neighborhood built environment and cognitive function of older persons: results from the Singapore longitudinal ageing study. Gerontology. 2018;64(2):149-156

19. Zijlema WL, Triguero-Mas M, Smith G, et al. The relationship between natural outdoor environments and cognitive functioning and its mediators. Environmental Research. 2017;155:268-275

20. Dadvand P, Pujol J, Macià D, et al. The association between lifelong greenspace exposure and 3-dimensional brain magnetic resonance imaging in Barcelona schoolchildren. Environmental Health Perspectives. 2018;126(2):027012

21. Markevych I, Tiesler CM, Fuertes E, et al. Access to urban green spaces and behavioural problems in children: Results from the GINIplus and LISAplus studies. Environment International. 2014;71:29-35

22. Ward JS, Duncan JS, Jarden A, et al. The impact of children's exposure to greenspace on physical activity, cognitive development, emotional wellbeing, and ability to appraise risk. Health \&Place. 2016;40:44-50

23. Tiesler CM, Birk M, Thiering E, et al. Exposure to road traffic noise and children's behavioural problems and sleep disturbance: results from the GINIplus and LISAplus studies. Environmental Research. 2013;123:1-8

24. Bodin T, Björk J, Ardö J, et al. Annoyance, sleep and concentration problems due to combined traffic noise and the benefit of quiet side. International Journal of Environmental Research and Public Health. 2015;12(2):16121628

25. Donovan GH, Michael YL, Gatziolis D, et al. Association between exposure to the natural environment, rurality, and attention-deficit hyperactivity disorder in children in New Zealand: a linkage study. The Lancet Planetary Health. 2019;3(5):e226-e234

26. Sharp W, Mangalmurti A, Hall C, et al. Associations between neighborhood, family factors and symptom change in childhood attention deficit hyperactivity disorder. Social Science and Medicine. 2019:112203.

27. Allen JG, MacNaughton P, Satish U, et al. Associations of cognitive function scores with carbon dioxide, ventilation, and volatile organic compound exposures in office workers: a controlled exposure study of green and conventional office environments. Environmental Health Perspectives. 2016;124(6):805-812

28. Gao T, Zhang T, Zhu L, et al. Exploring psychophysiological restoration and individual preference in the different environments based on virtual reality. International Journal of Environmental Research and Public Health. 2019;16(17):3102

29. Amoly E, Dadvand P, Forns J, et al. Green and blue spaces and behavioral development in Barcelona schoolchildren: the BREATHE project. Environmental Health Perspectives. 2014;122(12):1351-1358

30. Amicone G, Petruccelli I, De Dominicis S, et al. Green breaks: the restorative effect of the school environment's green areas on children's cognitive performance. Frontiers in Psychology. 2018;9:1579

31. Cherrie MP, Shortt NK, Mitchell RJ, et al. Green space and cognitive ageing: A retrospective life course analysis in the Lothian Birth Cohort 1936. Social Science and Medicine. 2018;196:56-65 
32. Dadvand P, Nieuwenhuijsen MJ, Esnaola M, et al. Green spaces and cognitive development in primary schoolchildren. Proceedings of the National Academy of Sciences. 2015;112(26):7937-7942

33. Crous-Bou M, Gascon M, Gispert JD, et al. Impact of urban environmental exposures on cognitive performance and brain structure of healthy individuals at risk for Alzheimer's dementia. Environment International. 2020:105546

34. Dadvand P, Tischer C, Estarlich $\mathrm{M}$, et al. Lifelong residential exposure to green space and attention: a population-based prospective study. Environmental Health Perspectives. 2017;125(9):097016

35. De Vries S, Ten Have M, van Dorsselaer S, et al. Local availability of green and blue space and prevalence of common mental disorders in the Netherlands. British Journal of Psychiatry Open. 2016;2(6):366-372

36. Besser LM, Rodriguez DA, McDonald N, et al. Neighborhood built environment and cognition in nondemented older adults: the multi-ethnic study of atherosclerosis. Social Science and Medicine. 2018;200:27-35

37. Kodama T, Morita K, Doi R, et al. Neurophysiological analyses in different color environments of cognitive function in patients with traumatic brain injury. Journal of Neurotrauma. 2010;27(9):1577-1584

38. Mytton OT, Townsend N, Rutter H, et al. Green space and physical activity: an observational study using Health Survey for England data. Health \&Place. 2012;18(5):1034-1041

39. Fernandes J, Arida RM, Gomez-Pinilla F. Physical exercise as an epigenetic modulator of brain plasticity and cognition. Neuroscience and Biobehavioral Reviews. 2017;80:443-456

40. Beyer KM, Kaltenbach A, Szabo A, et al. Exposure to neighborhood green space and mental health: evidence from the survey of the health of Wisconsin. International Journal of Environmental Research and Public Health. 2014;11(3):3453-3472

41. Lukasik KM, Waris $\mathrm{O}$, Soveri A, et al. The relationship of anxiety and stress with working memory performance in a large non-depressed sample. Frontiers in Psychology. 2019;10:4.

42. Montello DR. Spatial cognition and architectural space: Research perspectives. Architectural Design. 2014;84(5):74-79

43. Roupé M. Development and implementations of virtual reality for decision-making in urban planning and building design: Chalmers Tekniska Hogskola (Sweden); 2013.

44. Kar AZ, Sarkar A. Exploring the role of Environment-Behavior Studies (EBS) in Residential ArchitectureFrom Literature Review to Field Study. International Research Journal of Engineering and Technology. 2017;4(11):409-418

45. Barton J, Rogerson M. The importance of greenspace for mental health. British Journal of Psychiatry International. 2017;14(4):79-81 